\title{
Comparative Analysis of Methods for Regularizing an Initial Boundary Value Problem for the Helmholtz Equation
}

\author{
Sergey Igorevich Kabanikhin, ${ }^{1,2}$ M. A. Shishlenin, ${ }^{2,3}$ D. B. Nurseitov, \\ A. T. Nurseitova, ${ }^{5}$ and S. E. Kasenov ${ }^{4}$ \\ ${ }^{1}$ Institute of Computational Mathematics and Mathematical Geophysics, Akademika Lavrentjeva, No. 6, Novosibirsk 630090, Russia \\ ${ }^{2}$ Novosibirsk State University, Pirogova Street 2, Novosibirsk 630090, Russia \\ ${ }^{3}$ Sobolev Institute of Mathematics, 4 Academy Koptyug Avenue, Novosibirsk 630090, Russia \\ ${ }^{4}$ National Open Research Laboratory of Information and Space Technologies, Kazakh National Technical University after K.I. Satpaev, \\ Seifullin Street 122/22, Almaty 050013, Kazakhstan \\ ${ }^{5}$ Kazakh National Pedagogical University Abai, 13 Dostyk Avenue, Almaty 050010, Kazakhstan
}

Correspondence should be addressed to S. E. Kasenov; syrym.kasenov@mail.ru

Received 10 April 2014; Revised 16 July 2014; Accepted 22 July 2014; Published 8 September 2014

Academic Editor: D. R. Sahu

Copyright (C) 2014 Sergey Igorevich Kabanikhin et al. This is an open access article distributed under the Creative Commons Attribution License, which permits unrestricted use, distribution, and reproduction in any medium, provided the original work is properly cited.

\begin{abstract}
We consider an ill-posed initial boundary value problem for the Helmholtz equation. This problem is reduced to the inverse continuation problem for the Helmholtz equation. We prove the well-posedness of the direct problem and obtain a stability estimate of its solution. We solve numerically the inverse problem using the Tikhonov regularization, Godunov approach, and the Landweber
\end{abstract} iteration. Comparative analysis of these methods is presented.

\section{Introduction}

Let us consider the initial boundary value problem (continuation problem) for the Helmholtz equation in the domain $\Omega=(0, l) \times(0, \pi)$ :

$$
\begin{gathered}
u_{x x}+u_{y y}+k^{2} u=0, \quad(x, y) \in \Omega, \\
u_{x}(0, y)=0, \quad y \in[0, \pi], \\
u(0, y)=f(y), \quad y \in[0, \pi], \\
u_{y}(x, 0)=u_{y}(x, \pi)=0, \quad x \in[0, l],
\end{gathered}
$$

where $k$ is a given constant. To find the function $u(x, y)$ in $\Omega$ from $f(y)$ is required.

The continuation problem is ill-posed problem; its solution is unique, but it does not depend continuously on the Cauchy data [1-10]. Note that the problem was studied by many authors. For example, Tuan and Quan [11] considered the case $0<k<1$ and proposed a regularization technique which allows one to obtain a stable solution in a two-dimensional domain. Regińska and Regiński [12] showed that if $k$ satisfies a certain condition, then the Cauchy problem for the Helmholtz equation has a stable solution in a three-dimensional domain. Isakov and Kindermann [13] used the singular value decomposition to prove that in a simple domain the considered problem becomes more stable with increasing $k$. The same result was obtained numerically for the general case. The uniqueness of the solution of the investigated problem was proved, for example, by Arendt and Regińska [14], where the concept of weak normal derivative was introduced in formulating the problem. In $[15,16]$ singular values of the continuation problem were obtained for the two-dimensional Helmholtz equation with complex wave number for simple geometry.

We consider two approaches to the numerical solution of the problem (1). The first consists of formulating problem (1) in an operator form $\mathscr{A} q=f$ and minimizing the coast functional $J(q)=\langle\mathscr{A} q-f, \mathscr{A} q-f\rangle$ by the Landweber iteration [7]. In the second approach, problem (1) is reduced to the system of linear algebraic equations which is solved 
using the Tikhonov regularization and Godunov approach. In this work we present a comparative analysis of the proposed methods for the numerical solution of the problem (1).

\section{The Direct and Inverse Problems}

Let us consider the direct (well-posed) problem of finding the function $u(x, y)$ from the relations

$$
\begin{gathered}
u_{x x}+u_{y y}+k^{2} u=0, \quad(x, y) \in \Omega, \\
u_{x}(0, y)=0, \quad y \in[0, \pi], \\
u(l, y)=q(y), \quad y \in[0, \pi], \\
u_{y}(x, 0)=u_{y}(x, \pi)=0, \quad x \in[0, l]
\end{gathered}
$$

and note that the continuation problem (1) can be reduced to the inverse problem of finding function $q(y)$ from (2)-(5) using the additional information

$$
u(0, y)=f(y), \quad y \in[0, \pi] .
$$

Let us consider some theoretical results $[7,17]$.

Definition 1. A function $u \in L_{2}(\Omega)$ is called a generalized solution of the direct problem (2)-(5), if for any $\omega \in H^{2}(\Omega)$ such that

$$
\begin{gathered}
\omega_{x}(0, y)=0, \quad y \in[0, \pi], \\
\omega(l, y)=0, \quad y \in[0, \pi], \\
\omega_{y}(x, 0)=\omega_{y}(x, \pi)=0, \quad x \in[0, l],
\end{gathered}
$$

the following equality holds:

$$
\begin{gathered}
\int_{0}^{l} \int_{0}^{\pi} u \cdot\left(\omega_{x x}+\omega_{y y}+k^{2} \omega\right) d y d x \\
-\int_{0}^{\pi} q(y) \omega_{x}(l, y) d y=0 .
\end{gathered}
$$

Theorem 2 (existence of a generalized solution of the direct problem). If $q \in L_{2}(0, \pi)$ and $k^{2} l^{2}<1$, then the direct problem (2)-(5) has a unique generalized solution $u \in L_{2}(\Omega)$ and the following estimate is true:

$$
\|u\|_{L_{2}(\Omega)} \leq\|q\|_{L_{2}(0, \pi)} \frac{\sqrt{l}}{1-k^{2} l^{2}} .
$$

Proof. Let us introduce the auxiliary problem

$$
\begin{gathered}
\omega_{x x}+\omega_{y y}=\bar{u}, \quad(x, y) \in \Omega, \\
\omega_{x}(0, y)=0, \quad y \in[0, \pi], \\
\omega(l, y)=0, \quad y \in[0, \pi], \\
\omega_{y}(x, 0)=\omega_{y}(x, \pi)=0, \quad x \in[0, l] .
\end{gathered}
$$

Integrating the identity

$$
\omega \bar{u}=\omega\left(\omega_{x x}+\omega_{y y}\right)=\left(\omega_{x} \omega\right)_{x}-\omega_{x}^{2}+\left(\omega_{y} \omega\right)_{y}-\omega_{y}^{2}
$$

over the domain $\Omega$ and considering (10)-(13), we obtain

$$
\begin{aligned}
\int_{0}^{l} \int_{0}^{\pi} \omega \bar{u} d y d x= & \left.\int_{0}^{\pi} \omega_{x} \omega\right|_{0, y} ^{l, y} d y+\left.\int_{0}^{l} \omega_{y} \omega\right|_{x, 0} ^{x, \pi} d x \\
& -\int_{0}^{l} \int_{0}^{\pi}\left(\omega_{x}^{2}+\omega_{y}^{2}\right) d y d x
\end{aligned}
$$

whence

$$
\int_{0}^{l} \int_{0}^{\pi}\left(\omega_{x}^{2}+\omega_{y}^{2}\right) d y d x \leq\|\omega\|_{L_{2}(\Omega)} \cdot\|u\|_{L_{2}(\Omega)} .
$$

Taking into account (12) and the equality

$$
\begin{gathered}
\omega(l, y)=\omega(x, y)+\int_{x}^{l} \omega_{\xi}(\xi, y) d \xi \\
|\omega(x, y)|^{2}=\int_{x}^{l} \omega_{\xi}^{2}(\xi, y) d \xi \leq \int_{0}^{l} 1 \cdot \omega_{\xi}^{2}(\xi, y) d \xi \\
\leq \int_{0}^{l} 1 d \xi \cdot \int_{0}^{l} \omega_{\xi}^{2}(\xi, y) d \xi=l \cdot \int_{0}^{l} \omega_{\xi}^{2}(\xi, y) d \xi
\end{gathered}
$$

we have

$$
\begin{gathered}
|\omega(x, y)|^{2} \leq l \cdot \int_{0}^{l} \omega_{\xi}^{2}(\xi, y) d \xi \\
\int_{0}^{l} \int_{0}^{\pi}|\omega(x, y)|^{2} d y d x \leq \int_{0}^{l} \int_{0}^{\pi}\left(l \cdot \int_{0}^{l} \omega_{\xi}^{2}(\xi, y) d \xi\right) d y d x \\
\|\omega\|_{L_{2}(\Omega)} \leq l^{2} \cdot\left\|\omega_{\xi}\right\|_{L_{2}(\Omega)} .
\end{gathered}
$$

Combining (16) and (18) yields

$$
\begin{gathered}
\left\|\omega_{x}\right\|_{L_{2}(\Omega)} \leq l \cdot\|\bar{u}\|_{L_{2}(\Omega)}, \\
\|\omega\|_{L_{2}(\Omega)} \leq l^{2} \cdot\|\bar{u}\|_{L_{2}(\Omega)} .
\end{gathered}
$$

From the identities

$$
\begin{aligned}
\omega_{x} \bar{u} & =\omega_{x}\left(\omega_{x x}+\omega_{y y}\right)=\frac{1}{2}\left(\omega_{x}^{2}\right)_{x}+\omega_{x} \omega_{y y} \\
& =\frac{1}{2}\left(\omega_{x}^{2}\right)_{x}+\left(\omega_{x} \omega_{y}\right)_{y}-\frac{1}{2}\left(\omega_{y}^{2}\right)_{x},
\end{aligned}
$$

it follows that

$$
\frac{1}{2}\left(\omega_{x}^{2}\right)_{x}+\left(\omega_{x} \omega_{y}\right)_{y}=\omega_{x} \bar{u}+\frac{1}{2}\left(\omega_{y}^{2}\right)_{x} .
$$

Integrating (21) on $\Omega$, we get

$$
\begin{aligned}
\frac{1}{2} \int_{0}^{\pi} \omega_{x}^{2}(l, y) d y= & \int_{0}^{l} \int_{0}^{\pi} \omega_{x} \bar{u} d y d x+\frac{1}{2} \int_{0}^{\pi} \omega_{y}^{2}(l, y) d y \\
& -\frac{1}{2} \int_{0}^{\pi} \omega_{y}^{2}(0, y) d y \\
\leq & \left\|\omega_{x}\right\|_{L_{2}(\Omega)} \cdot\|\bar{u}\|_{L_{2}(\Omega)} \leq l\|\bar{u}\|_{L_{2}(\Omega)}^{2} .
\end{aligned}
$$


Due to (8), we obtain

$$
\begin{aligned}
& \int_{0}^{l} \int_{0}^{\pi}\left(\bar{u}^{2}+k^{2} \omega \bar{u}\right) d y d x \\
& \quad \leq\|q(y)\|_{L_{2}(0, \pi)} \cdot\left\|\omega_{x}(l, y)\right\|_{L_{2}(0, \pi)} .
\end{aligned}
$$

Hence,

$$
\begin{aligned}
&\|\bar{u}\|_{L_{2}(\Omega)}^{2} \leq\|q(y)\| \cdot\left\|\omega_{x}(l, y)\right\|+k^{2}\|\omega\| \cdot\|\bar{u}\| \\
& \leq\|q(y)\| \cdot \sqrt{l}\|\bar{u}\|+k^{2}\|\bar{u}\| \cdot l^{2}\|\bar{u}\|, \\
&\|\bar{u}\|_{L_{2}(\Omega)} \leq\|q(y)\| \cdot \frac{\sqrt{l}}{1-k^{2} l^{2}} .
\end{aligned}
$$

Thus, we have proved the well-posedness of the direct problem, which allows us to apply well-elaborated computational methods. Also, a stability estimate has been obtained in $L_{2}$.

\section{Landweber Iteration}

3.1. Formulation in the Operator Form and Description of the Algorithm. Let us reduce the inverse problem (2)-(6) to the operator equation. Let us consider the operator $\mathscr{A}$ such that

$$
\mathscr{A}: q(y) \longmapsto u(0, y),
$$

where $u(x, y)$ is the solution of the direct problem (2)-(5). Then the inverse problem (2)-(6) takes the form

$$
\mathscr{A} q=f .
$$

We will find the solution of the problem (26) by minimizing the following functional $[7,18-20]$ :

$$
J(q)=\|\mathscr{A} q-f\|^{2}=\int_{0}^{\pi}[u(0, y ; q)-f(y)]^{2} d y
$$

using the Landweber iteration

$$
q_{n+1}=q_{n}-\alpha J^{\prime} q_{n},
$$

where $\alpha \in\left(0,1 /\|\mathscr{A}\|^{2}\right)$ is the descent parameter [7].

Let us describe the iterative algorithm. First we choose the initial approximation $q_{0}$ and we suppose that we calculated successively $q_{n}$ by formula (28). Assuming that we have found $q_{n}$, we show below how to calculate $q_{n+1}$.

(1) Solve the direct problem (2)-(5) with the known $q_{n}$.

(2) Calculate $J\left(q_{n}\right)$ by formula (27).

(3) Check the stopping criterion $J\left(q_{n}\right) \leq \eta \varepsilon^{2}$ [21]. Finish if $q_{n}$ meets this inequality.

(4) Solve the adjoint problem

$$
\begin{gathered}
\psi_{x x}+\psi_{y y}+k^{2} \psi=0, \quad(x, y) \in \Omega, \\
\psi(l, y)=0, \quad y \in[0, \pi], \\
\psi_{x}(0, y)=2(u(0, y)-f(y)), \quad y \in[0, \pi], \\
\psi_{y}(x, \pi)=\psi_{y}(x, 0)=0, \quad x \in[0, l] .
\end{gathered}
$$

(5) Calculate the gradient $J^{\prime}\left(q_{n}\right)$ by the formula

$$
J^{\prime} q=\psi_{x}(l, y) .
$$

(6) Calculate the next approximation $q_{n+1}=q_{n}-\alpha J^{\prime} q_{n}$ and proceed to step 1 .

3.2. The Numerical Solution of the Direct and Adjoint Problems. The direct and adjoint problems are solved using the direct finite-difference method. For discretizing the direct problem, we construct a grid in $\Omega$ with steps $h_{x}=l / N_{x}, h_{y}=$ $\pi / N_{y}$, where $N_{x}, N_{y}$ are positive integers. Let us denote the grid by $\omega_{h}=\left\{x=i h_{x}, y=j h_{y} ; i=\overline{0, N_{x}}, j=\overline{0, N_{y}}\right\}$. After exchanging derivatives by finite-difference analoges with the second order, we obtain the following discrete direct problem (2)-(5):

$$
\begin{gathered}
\frac{u_{i+1, j}-2 u_{i, j}+u_{i-1, j}}{h_{x}^{2}}+\frac{u_{i, j+1}-2 u_{i, j}+u_{i, j-1}}{h_{y}^{2}}+k^{2} u_{i, j}=0, \\
i=\overline{1, N_{x}-1}, \quad j=\overline{1, N_{y}-1}, \\
\frac{u_{1, j}-u_{0, j}}{h_{x}}=0, \quad j=\overline{1, N_{y}-1}, \\
u_{N_{x}, j}=q_{j}, \quad j=\overline{1, N_{y}-1}, \\
\frac{u_{i, 1}-u_{i, 0}}{h_{y}}=\frac{u_{i, N_{x}}-u_{i, N_{x}-1}}{h_{y}}=0, \quad i=\overline{0, N_{x}} .
\end{gathered}
$$

By introducing the parameters $a=1 / h_{x}^{2}, b=1 / h_{y}^{2}, c=k^{2}-$ $2 a-2 b$, we get

$$
\begin{array}{r}
a u_{i-1, j}+b u_{i, j-1}+c u_{i, j}+b u_{i, j+1}+a u_{i+1, j}=0, \\
i=\overline{1, N_{x}-1}, \quad j=\overline{1, N_{y}-1}, \\
u_{1, j}-u_{0, j}=0, \quad j=\overline{1, N_{y}-1}, \\
u_{N_{x}, j}=q_{j}, \quad j=\overline{1, N_{y}-1}, \\
u_{i, 1}-u_{i, 0}=u_{i, N_{x}}-u_{i, N_{x}-1}=0, \quad i=\overline{0, N_{x}} .
\end{array}
$$

Thus, we obtain the system of algebraic equations

$$
A_{T} X_{T}=B_{T},
$$

where $A_{T}$ is a matrix of size $\left(N_{x}+1\right)\left(N_{y}+1\right), X_{T}$ is an unknown vector of the form

$$
\begin{array}{r}
X_{T}=\left(u_{0,0}, u_{0,1}, u_{0,2}, \ldots, u_{0, N_{y}}, u_{1,0}, u_{1,1}, u_{1,2}, \ldots,\right. \\
\left.u_{1, N_{y}}, \ldots, u_{N_{x}, 0}, u_{N_{x}, 1}, u_{N_{x}, 2}, \ldots, u_{N_{x}, N_{y}}\right),
\end{array}
$$

and $B_{T}$ is the data vector (boundary and additional conditions). 
Similarly, the discrete adjoint problem (29) has the form

$$
\begin{array}{r}
a \psi_{i-1, j}+b \psi_{i, j-1}+c \psi_{i, j}+b \psi_{i, j+1}+a \psi_{i+1, j}=0, \\
i=\overline{1, N_{x}-1,} \quad j=\overline{1, N_{y}-1}, \\
\psi_{1, j}-\psi_{0, j}=2\left(u_{0, j}-f_{j}\right), \quad j=\overline{1, N_{y}-1}, \\
\psi_{N_{x}, j}=0, \quad j=\overline{1, N_{y}-1}, \\
\psi_{i, 1}-\psi_{i, 0}=\psi_{i, N_{x}}-\psi_{i, N_{x}-1}=0, \quad i=\overline{0, N_{x}} .
\end{array}
$$

As above, this problem can be reformulated in a matrix form

$$
A_{T} Y_{T}=\bar{B}_{T}
$$

where

$$
\begin{array}{r}
Y_{T}=\left(\psi_{0,0}, \psi_{0,1}, \psi_{0,2}, \ldots, \psi_{0, N_{y}}, \psi_{1,0}, \psi_{1,1}, \psi_{1,2}, \ldots,\right. \\
\left.\psi_{1, N_{y}}, \ldots, \psi_{N_{x}, 0}, \psi_{N_{x}, 1}, \psi_{N_{x}, 2}, \ldots, \psi_{N_{x}, N_{y}}\right)
\end{array}
$$

is an unknown vector and $\bar{B}_{T}$ is the data vector (boundary and additional conditions of the adjoint problem).

3.3. Results of the Numerical Experiment. Let $l=1, N_{x}=$ $N_{y}=20$. We choose the parameter $k=0.9$. In order to test the algorithm, we assume that the exact solution has the form $q(y)=1-\cos (2 y)$ and calculate the corresponding additional information $f$. Then, let $q_{0}(y)=0.1$ be the initial approximation; we try to restore the original exact solution using the Landweber iteration with $\alpha=0.01$. If the data are given with an error $\varepsilon$, we choose the following one as a stopping criterion: $J\left(q_{n}\right) \leq \eta \varepsilon^{2}[21]$.

The computational experiment was carried out for different noise levels. Tables 1, 2, and 3 show the calculation results obtained using PC Intel(R)Core(TM) i7 processor with a frequency $3.9 \mathrm{GHz}$.

The approximate solution in the case of $\varepsilon=0.05$ is shown in Figure 1.

We observe that in the case of no noise the functional $J(q)$ decreases monotonically, while in the other cases the decrease stops after 100 iterations. This phenomenon can be explained by the error that arises in solving the direct and adjoint problems. Note that in the case of noise, the stopping criterion does not guarantee the minimal error in the solution of the inverse problem. However, the criterion ensures that the error is of the same order as that of the minimal one, since further calculation leads to an increase in the error.

\section{Regularization Methods}

In this section we consider a discrete analog of problem (1) and study the stability of its solution. Tikhonov regularization method and Godunov approach method are applied.

4.1. The Discretization of Problem (1). We reduce the continuation problem (1) to the system of linear algebraic equations as follows [22]:

$$
A X=B
$$

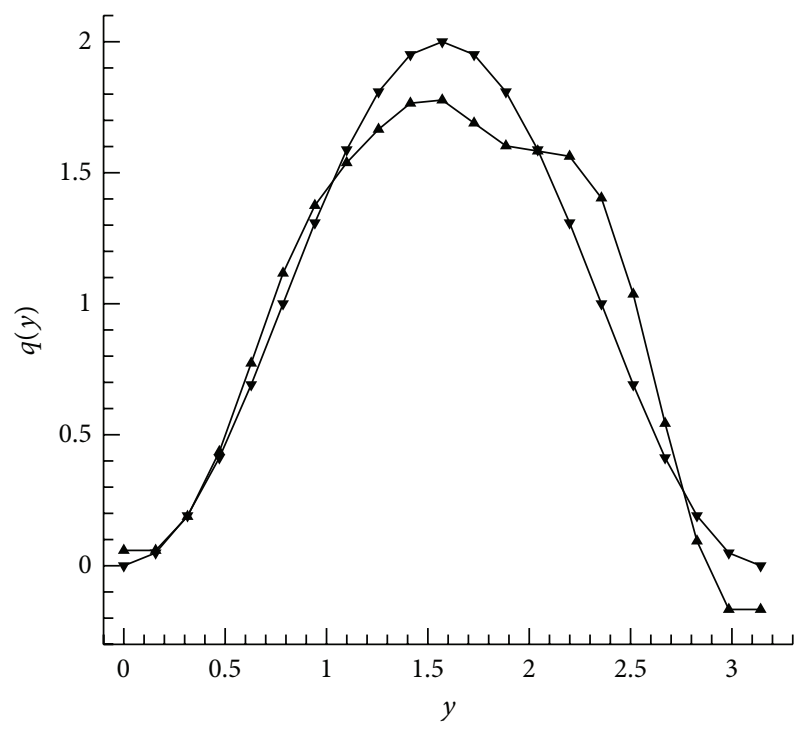

FIGURE 1: $\boldsymbol{\nabla}$ indicates exact solution; $\boldsymbol{\Delta}$ indicates solution obtained by the Landweber iteration.

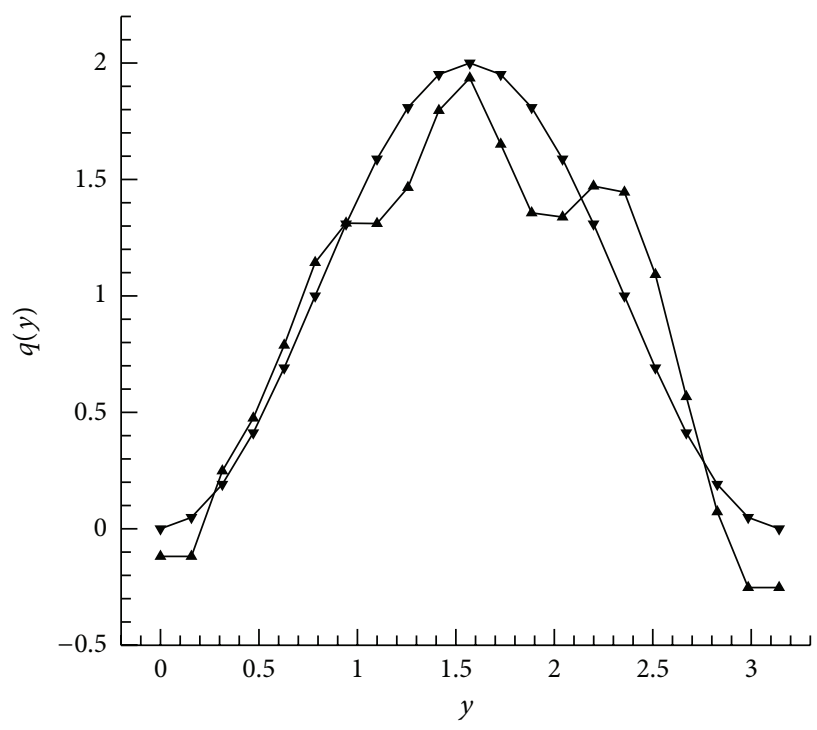

Figure 2: $\boldsymbol{\nabla}$ indicates exact solution $q(y)$; $\boldsymbol{\Delta}$ indicates solution obtained by the Tikhonov regularization.

where $A$ is a matrix of size $\left(N_{x}+1\right)\left(N_{y}+1\right), B$ is the data vector, and $X$ is the desired vector of the form

$$
\begin{array}{r}
X=\left(u_{0,0}, u_{0,1}, u_{0,2}, \ldots, u_{0, N_{y}}, u_{1,0}, u_{1,1}, u_{1,2}, \ldots,\right. \\
\left.u_{1, N_{y}}, \ldots, u_{N_{x}, 0}, u_{N_{x}, 1}, u_{N_{x}, 2}, \ldots, u_{N_{x}, N_{y}}\right) .
\end{array}
$$

Assuming $l=1, N_{x}=N_{y}=50, k=0.9$, and $q(y)=$ $1-\cos (2 y)$, we calculate the norm and the condition number $\mu(A)$ of the matrices $A$ and $A_{T}$ corresponding to the original problem (1) and the direct problem (2)-(5), respectively.

The matrix $A$ is ill-conditioned [23] (see decreasing of its singular values in Figure 4 ). The condition number and matrix norma of the discrete direct problem are presented in 
TABLE 1: Calculation results in the case of no noise $\varepsilon=0$.

\begin{tabular}{lcccc}
\hline Number & The number of iterations $n$ & $\left\|q_{T}-\tilde{q}\right\|$ & $J(q)$ & Runtime \\
\hline 1 & 10 & 0.8243 & 0.094 & $4 \mathrm{sec}$ \\
2 & 100 & 0.0632 & $9.42 \cdot 10^{-5}$ & $40 \mathrm{sec}$ \\
3 & 1000 & 0.0414 & $9.55 \cdot 10^{-7}$ & $6 \mathrm{~min} 40 \mathrm{sec}$ \\
4 & 5000 & 0.0311 & $1.74 \cdot 10^{-8}$ & $33 \mathrm{~min} 30 \mathrm{sec}$ \\
5 & 7318 & 0.0304 & $9.29 \cdot 10^{-9}$ & $49 \mathrm{~min}$ \\
\hline
\end{tabular}

TABLE 2: Calculation results in the case of noise within $1 \% \varepsilon=0.01$.

\begin{tabular}{lcccc}
\hline Number & The number of iterations $n$ & $\left\|q_{T}-\tilde{q}\right\|$ & $J(q)$ & \\
\hline 1 & 10 & 0.83 & 0.09 & \\
2 & 100 & 0.077 & $4.91 \cdot 10^{-4}$ & $4 \mathrm{sec}$ \\
3 & 1000 & 0.047 & $3.38 \cdot 10^{-4}$ & \\
4 & 1508 & 0.051 & $3.32 \cdot 10^{-4}$ & \\
\hline
\end{tabular}

TABLE 3: Calculation results in the case of noise within $5 \% \varepsilon=0.05$.

\begin{tabular}{lcccc}
\hline Number & The number of iterations $n$ & $\left\|q_{T}-\tilde{q}\right\|$ & $J(q)$ & Runtime \\
\hline 1 & 10 & 0.86 & $9.07 \cdot 10^{-2}$ & $4 \mathrm{sec}$ \\
2 & 100 & 0.165 & $9.20 \cdot 10^{-3}$ & $40 \mathrm{sec}$ \\
3 & 1000 & 0.210 & $8.33 \cdot 10^{-3}$ & $6 \mathrm{~min} 32 \mathrm{sec}$ \\
4 & 2000 & 0.297 & $8.15 \cdot 10^{-3}$ & $13 \mathrm{~min} 4 \mathrm{sec}$ \\
5 & 2418 & 0.326 & $8.10 \cdot 10^{-3}$ & $15 \mathrm{~min} 48 \mathrm{sec}$ \\
\hline
\end{tabular}

TABLE 4: The characteristics of the matrices $A$ and $A_{T}$.

\begin{tabular}{lcc}
\hline Matrix & $\mu(A)$ & $\|A\|$ \\
\hline$A$ & $6.02 \cdot 10^{17}$ & 321098.0 \\
$A_{T}$ & $3.42 \cdot 10^{6}$ & 321098.0 \\
\hline
\end{tabular}

Table 4 and Figure 5. We see that the direct problem is wellposed.

In view of the ill-conditioning of the matrix $A$, that is, the ill-posedness of the original problem, we will use regularization methods.

4.2. Tikhonov Regularization. The Tikhonov regularization consists of replacing the system $A X=B$ by the system $(\alpha I+$ $\left.A^{*} A\right) \widetilde{X}=A^{*} B[7]$. We choose the regularization parameter $\alpha$, minimizing the discrepancy $\left\|X_{\alpha_{i}}\right\|-\left\|X_{\alpha_{i-1}}\right\|$ according to [24].

As above, we put $N_{x}=N_{y}=20, k=0.9$, and $q(y)=$ $1-\cos (2 y)$ and calculate with $\varepsilon=0, \varepsilon=0.01$, and $\varepsilon=0.05$. The approximate solution in the case of $\varepsilon=0.05$ is shown in Figure 2.

4.3. Godunov Approach. S. K. Godunov proposed considering the extended system

$$
\left[\begin{array}{c}
(1-\alpha) A \\
\alpha D
\end{array}\right] X=\left[\begin{array}{c}
(1-\alpha) f \\
0
\end{array}\right]
$$

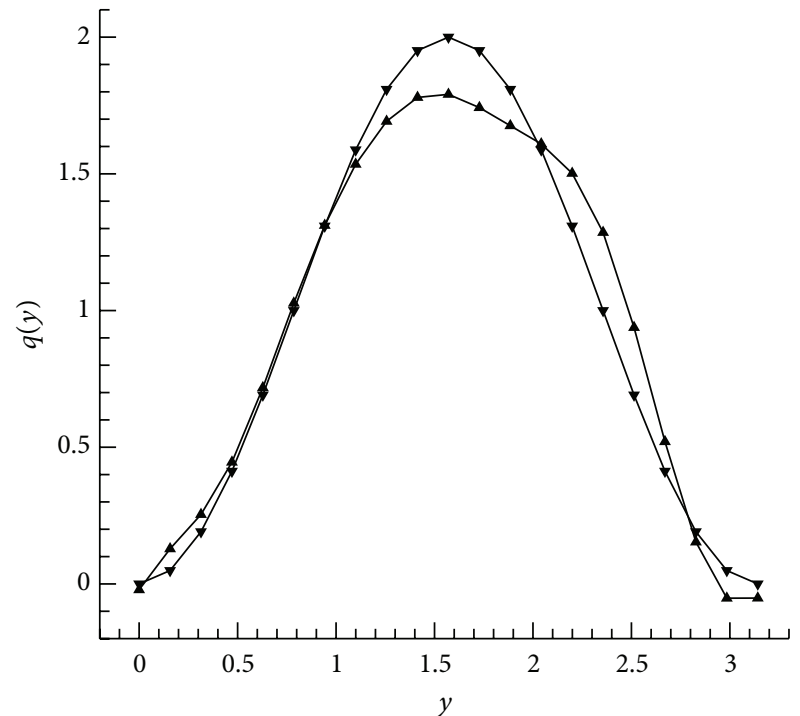

FIGURE 3: $\boldsymbol{\nabla}$ indicates exact solution $q(y)$; $\boldsymbol{\Delta}$ indicates solution obtained by the Godunov regularization.

whereas $D$ contains some a priori information concerning the inverse problem solution. We take as a priori information the existence of the second derivative of the solution [25].

We choose $\alpha$ to minimize $\left\|X_{\alpha_{i}}\right\|-\left\|X_{\alpha_{i-1}}\right\|$.

For $N_{x}=N_{y}=20, k=0.9$, and $q(y)=1-\cos (2 y)$, we calculate with different values of $\varepsilon$. The result is shown in Figure 3. 


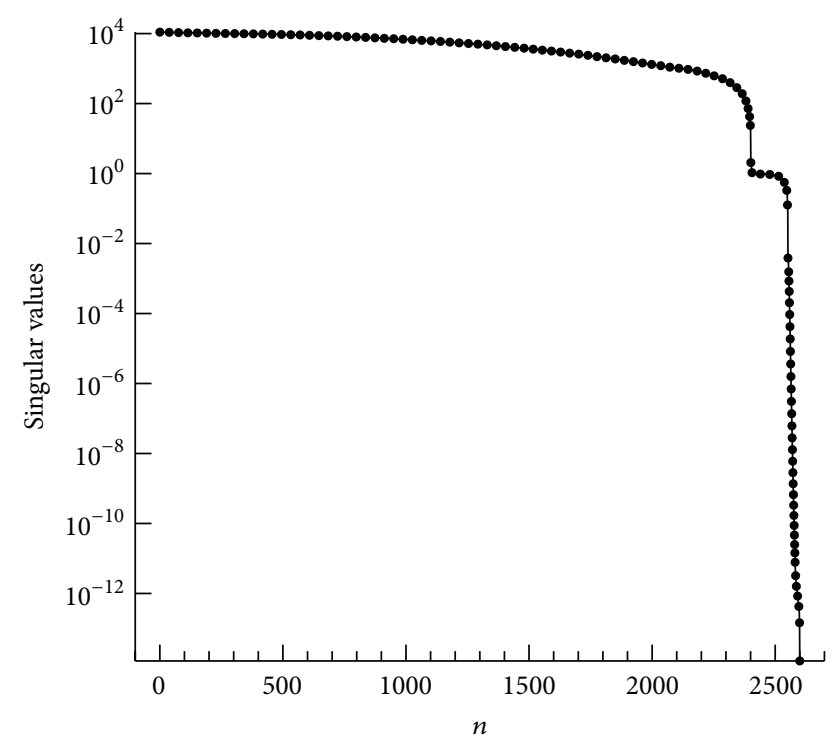

FIgURE 4: Singular values of matrix $A$ for $N=50$.

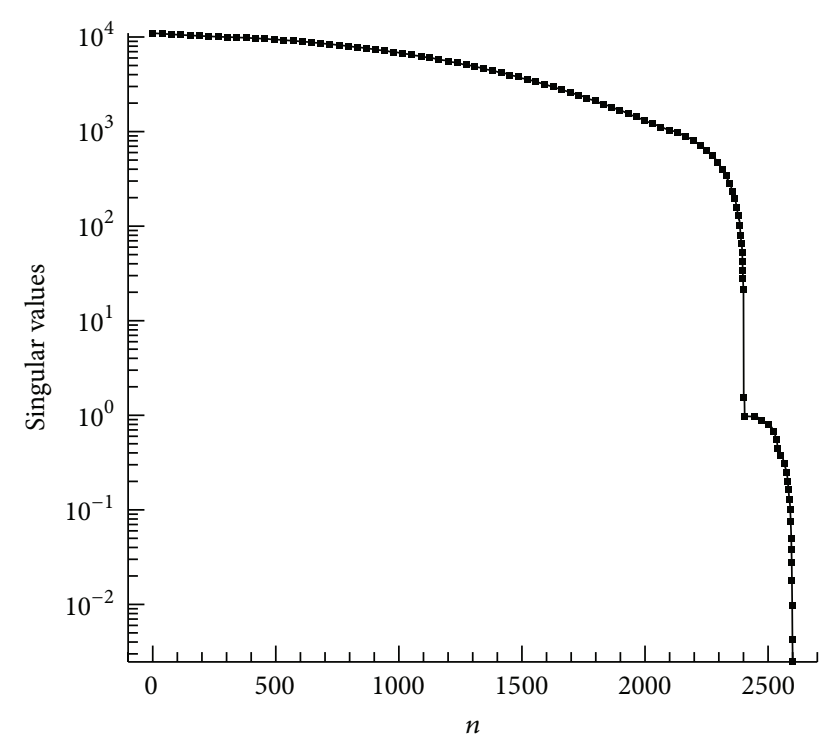

Figure 5: Singular values of matrix $A_{T}$ for $N=50$.

TABLE 5: Comparative analysis of methods in the case of no noise.

\begin{tabular}{lccc}
\hline Number & Name methods & $\left\|q_{T}-\tilde{q}\right\|$ & Runtime \\
\hline 1 & Landweber iteration & 0.030 & $49 \mathrm{~min}$ \\
2 & Tikhonov regularization & 0.021 & $23 \mathrm{sec}$ \\
3 & Godunov regularization & 0.019 & $46 \mathrm{sec}$ \\
\hline
\end{tabular}

\section{Comparative Analysis of Methods}

Tables 5, 6, and 7 present the results of the numerical solution of the problem (1) with different levels of noise. We see that the Godunov method is more accurate compared to the other methods. The same is demonstrated in Figure 6.

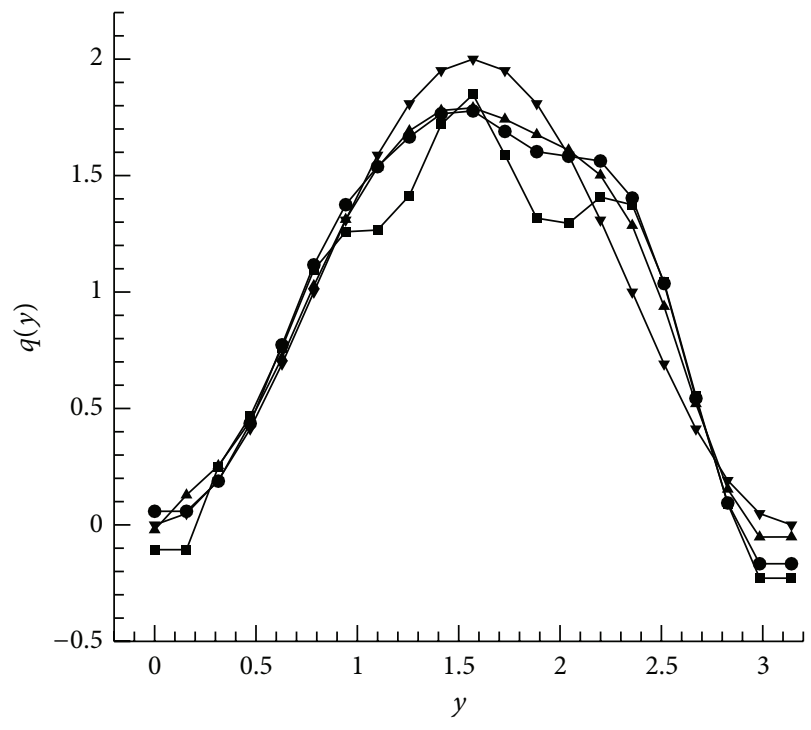

FIGURE 6: $\boldsymbol{\nabla}$ indicates exact solution $q(y)$; $\bullet$ indicates Landweber iteration; indicates Tikhonov regularization; $\boldsymbol{\Delta}$ indicates Godunov regularization.

TABLE 6: Comparative analysis of methods in the case of noise within $1 \%$.

\begin{tabular}{lccc}
\hline Number & Name methods & $\left\|q_{T}-\tilde{q}\right\|$ & Runtime \\
\hline 1 & Landweber iteration & 0.051 & $10 \mathrm{~min}$ \\
2 & Tikhonov regularization & 0.190 & $15 \mathrm{sec}$ \\
3 & Godunov regularization & 0.055 & $17 \mathrm{sec}$ \\
\hline
\end{tabular}

TABLE 7: Comparative analysis of methods in the case of noise within $5 \%$.

\begin{tabular}{lccc}
\hline Number & Name methods & $\left\|q_{T}-\tilde{q}\right\|$ & Runtime \\
\hline 1 & Landweber iteration & 0.5349 & $18 \mathrm{~min} 24 \mathrm{sec}$ \\
2 & Tikhonov regularization & 0.4088 & $7 \mathrm{sec}$ \\
3 & Godunov regularization & 0.2989 & $12 \mathrm{sec}$ \\
\hline
\end{tabular}

\section{Conflict of Interests}

The authors declare that there is no conflict of interests regarding the publication of this paper.

\section{Acknowledgments}

The work was partially supported by the Ministry of Education and Science of the Russian Federation, joint Project SB RAS and NAS of Ukraine, 2013, no. 12, and RFBR Grant 1401-00208.

\section{References}

[1] T. Delillo, V. Isakov, N. Valdivia, and L. Wang, "The detection of the source of acoustical noise in two dimensions," SIAM Journal on Applied Mathematics, vol. 61, no. 6, pp. 2104-2121, 2001.

[2] T. DeLillo, V. Isakov, and N. a. . Valdivia, "The detection of surface vibrations from interior acoustical pressure," Inverse Problems, vol. 19, no. 3, pp. 507-524, 2003. 
[3] W. S. Hall and X. Q. Mao, "A boundary element investigation of irregular frequencies in electromagnetic scattering," Engineering Analysis with Boundary Elements, vol. 16, no. 3, pp. 245-252, 1995.

[4] L. Marin, L. Elliott, P. J. Heggs, D. B. Ingham, D. Lesnic, and X. Wen, "An alternating iterative algorithm for the Cauchy problem associated to the Helmholtz equation," Computer Methods in Applied Mechanics and Engineering, vol. 192, no. 5-6, pp. 709722, 2003.

[5] T. Regińska and K. Regiński, "Approximate solution of a Cauchy problem for the Helmholtz equation," Inverse Problems, vol. 22, no. 3, pp. 975-989, 2006.

[6] J. Hadamard, Lectures on Cauchys Problem in Linear Partial Differential Equations, Yale University Press, New Haven, Conn, USA, 1923.

[7] S. I. Kabanikhin, Inverse and Ill-Posed Problems. Theory and Applications, vol. 55 of Inverse and Ill-Posed Problems Series, De Gruyter, Berlin, Germany, 2011.

[8] F. John, "Continuous dependence on data for solutions of partial differential equations with a presribed bound," Communications on Pure and Applied Mathematics, vol. 13, pp. 551-585, 1960.

[9] G. Alessandrini, L. Rondi, E. Rosset, and S. Vessella, "The stability for the Cauchy problem for elliptic equations," Inverse Problems, vol. 25, no. 12, Article ID 123004, 47 pages, 2009.

[10] S. I. Kabanikhin and A. L. Karchevsky, "Optimizational method for solving the Cauchy problem for an elliptic equation," Journal of Inverse and Ill-Posed Problems, vol. 3, no. 1, pp. 21-46, 1995.

[11] N. H. Tuan and P. H. Quan, "A Cauchy problem for Helmholtz equation: regularization and error estimates," Acta Universitatis Apulensis, no. 25, pp. 177-188, 2011.

[12] T. Regiñska and K. Regiñski, "A Cauchy problem for the Helmholtz equation: application to analysis of light propagation in solids," Tech. Rep. 06-4, Berichtsreihe des Mathematischen Seminars, Christian-Albrechts-University of Kiel, 2006.

[13] V. Isakov and S. Kindermann, "Subspaces of stability in the Cauchy problem for the Helmholtz equation," Methods and Applications of Analysis, vol. 18, no. 1, pp. 1-30, 2011.

[14] W. Arendt and T. Regińska, "An ill-posed boundary value problem for the Helmholtz equation on Lipschitz domains," Journal of Inverse and Ill-Posed Problems, vol. 17, no. 7, pp. 703-711, 2009.

[15] S. I. Kabanikhin and M. A. Shishlenin, "Regularization of the continuation problem for parabolic and elliptic equations from the part of the boundary," Eurasian Journal of Mathematical and Computer Applications, 2014.

[16] S. I. Kabanikhin, Y. S. Gasimov, D. B. Nurseitov, M. A. Shishlenin, B. B. Sholpanbaev, and S. Kasenov, "Regularization of the continuation problem for elliptic equations," Journal of Inverse and Ill-Posed Problems, vol. 21, no. 6, pp. 871-884, 2013.

[17] S. I. Kabanikhin, M. A. Bektemesov, and A. T. Nurseitova, Iteration Methods of Solving Inverse and Ill-Posed Problems with Data on the Part of the Boundary, Almaty-Novosibirsk, 2006, (Russian).

[18] S. I. Kabanikhin, D. B. Nurseitov, M. A. Shishlenin, and B. B. Sholpanbaev, "Inverse problems for the ground penetrating radar," Journal of Inverse and Ill-Posed Problems, vol. 21, no. 6, pp. 885-892, 2013.

[19] S. I. Kabanikhin and O. I. Krivorot'ko, "A numerical method for determining the amplitude of a wave edge in shallow water approximation," Applied and Computational Mathematics, vol. 12, no. 1, pp. 91-96, 2013.
[20] S. Kabanikhin, A. Hasanov, I. Marinin, O. Krivorotko, and D. Khidasheli, "A variational approach to reconstruction of an initial tsunami source perturbation," Applied Numerical Mathematics, vol. 83, pp. 22-37, 2014.

[21] U. Hamarik and R. Palm, "Comparison of stopping rules in conjugate gradient type methods for solving ill-posed problems," in Proceedings of the 10th International Conference MAthematical Modelling (MMA '05) and Analysis 2nd International Conference Computational Methods in Applied Mathematics, pp. 285-291, Technika, Trakai, Lithuania, 2005.

[22] M. A. Shishlenin, "Matrix method for discrete inverse source problem for acoustic equation," Siberian Electronic Mathematical Reports, vol. 11, pp. C.98-C.114, 2014.

[23] S. K. Godunov, Lectures on Modern Aspects of Linear Algebra, Science book, Novosibirsk, Russia, 2002.

[24] L. Wu, "A parameter choice method for Tikhonov regularization," Electronic Transactions on Numerical Analysis, vol. 16, pp. 107-128, 2003.

[25] S. K. Godunov, A. G. Antonov, O. P. Kiriljuk, and V. I. Kostin, Guaranteed Accuracy in Numerical Linear Algebra, Amsterdam, The Netherlands, Springer, 1993. 


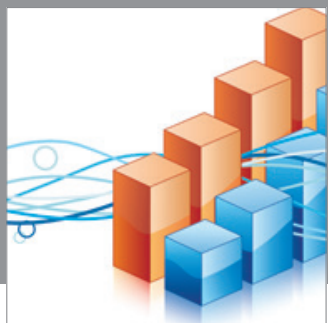

Advances in

Operations Research

mansans

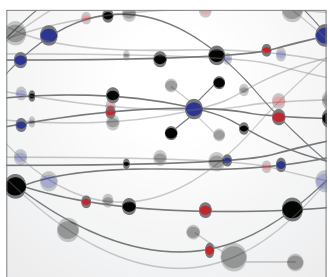

The Scientific World Journal
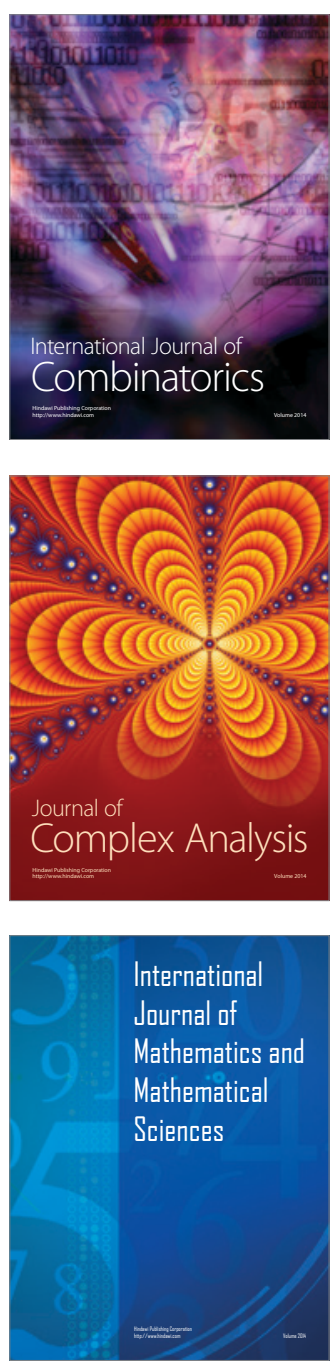


Submit your manuscripts at http://www.hindawi.com
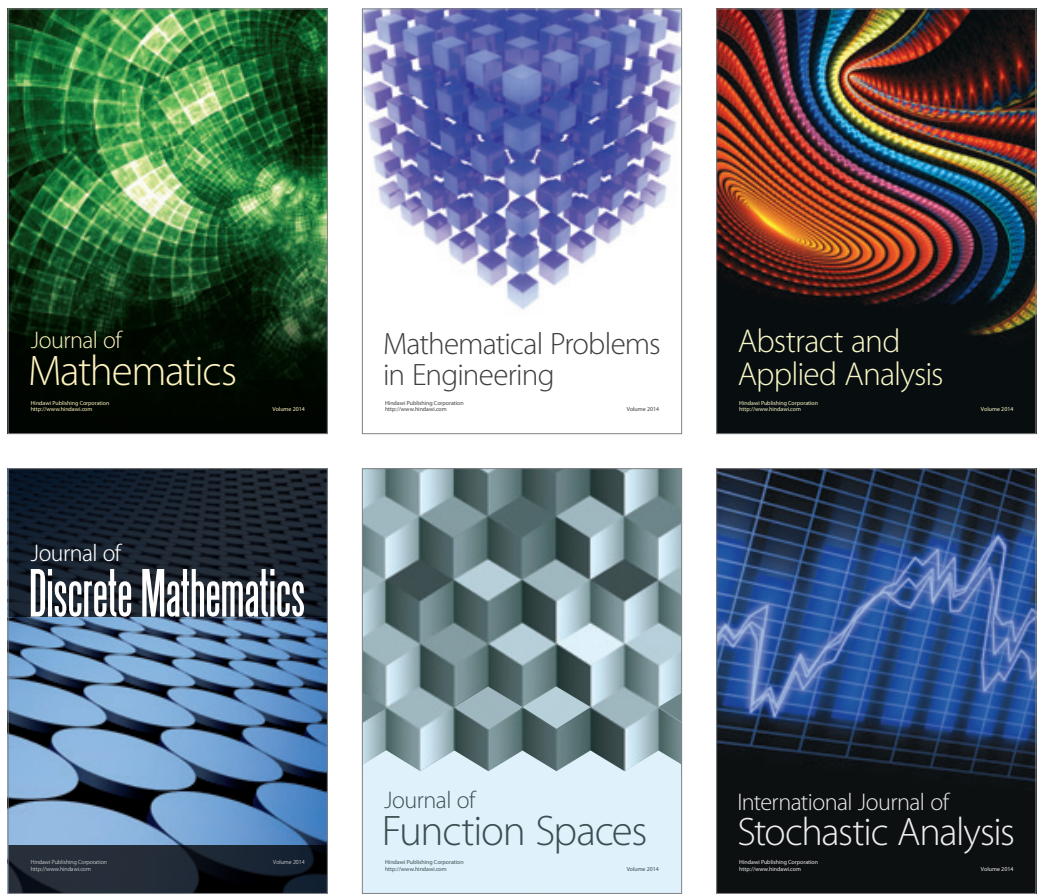

Journal of

Function Spaces

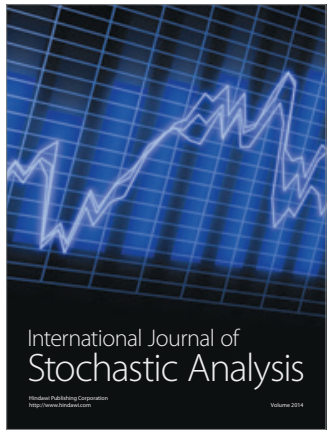

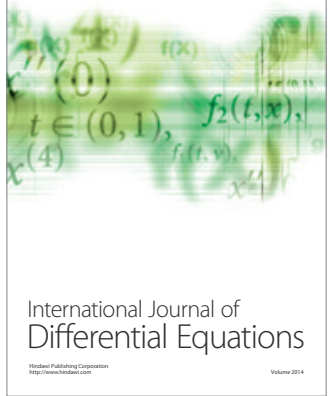
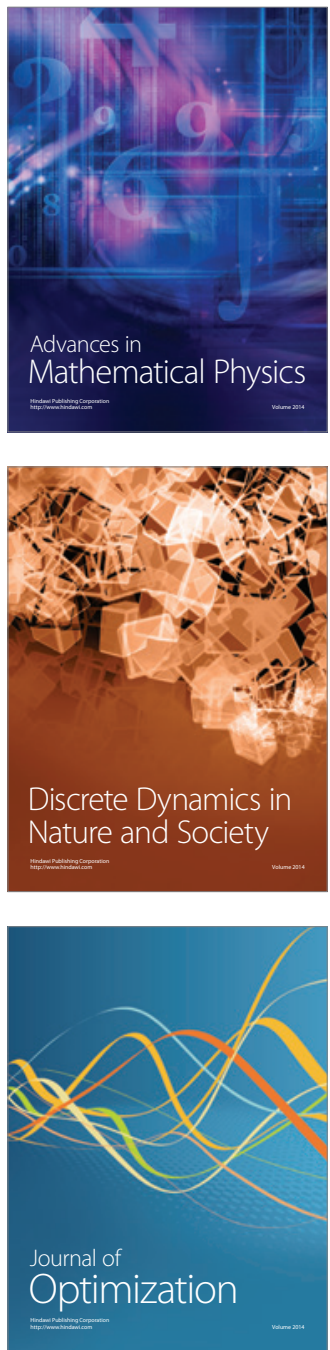